\title{
Imipramine plus cognitive behavioural therapy (CBT) was more effective than placebo plus CBT in adolescents with comorbid anxiety and depression who refused to attend school
}

Bernstein GA, Borchardt CM, Perwien AR, et al. Imipramine plus cognitive-behavioral therapy in the treatment of school refusal.J Am Acad Child Adolesc Psychiatry 2000 Mar;39:276-83.

QUESTION: In adolescents with comorbid anxiety and major depressive disorders who refused to attend school, what is the effectiveness of 8 weeks of imipramine plus cognitive behavioural therapy (CBT) compared with placebo plus CBT?

\section{Design}

Randomised \{allocation concealed*\} ${ }^{*}$, blinded (patients and outcome assessors)*, placebo controlled trial with 8 weeks of follow up.

\section{Setting}

Psychiatry Department, University of Minnesota, Minneapolis, USA.

\section{Patients \\ 63 postpubertal adolescents between 12 and 18 years of age (mean age $14 \mathrm{y}, 60 \%$ girls) who had a minimum of $20 \%$ days absent from school in the 4 weeks before assessment for the study, had a minimum of 1 anxiety disorder, and had a diagnosis of major depressive disor- der (MDD). The adolescents also had to have a minimum score of 5 on the Anxiety subscale of the Anxiety Rating for Children-Revised (ARC-R), and a minimum score of 35 on the Children's Depression Rat- ing Scale-Revised (CDRS-R). Exclusion criteria included diagnoses of attention deficit hyperactivity disorder, conduct disorder, bipolar disorder, eating disorder, or alcohol or drug abuse; contraindication to the study drug; current use of a psychotropic medication; and pregnancy.}

\section{Intervention}

31 patients were allocated to imipramine (gradual increases every $3-5$ days to $3 \mathrm{mg} / \mathrm{kg}$ /day by end of week 2) plus 8 sessions of CBT, and 32 patients to matching placebo plus CBT.

\section{Main outcome measures}

Weekly school attendance rates based on percentage of hours attended, and scores on anxiety and depression rating scales.

\footnotetext{
Main results

Analysis was by intention to treat. Over 8 weeks, there was a difference between the 2 groups on attendance after controlling for baseline attendance; the table shows mean weekly attendance in the eighth week. Of the anxiety and depression rating scales, only the CDRS-R showed differential improvement between groups, with depression decreasing at a faster rate for the imipramine group (table). Patients receiving imipramine were more likely to meet the attendance goal (weekly attendance of $75 \%$ of school hours or better) than were those receiving placebo (table).
}

\section{Conclusion}

Imipramine in combination with cognitive behavioural therapy (CBT) was more effective than placebo with CBT in helping adolescents with comorbid anxiety and major depressive disorders return to school and in decreasing depression over 8 weeks of treatment.

*See glossary.

$\dagger$ Information provided by author.

\author{
Sources of funding: \\ National Institute of \\ Mental Health; \\ imipramine and \\ placebo provided by \\ Biocraft Laboratories, \\ Inc;pill containers \\ provided by Apothecary \\ Products, Inc; an ECG \\ machine was from a \\ Marquette Electronics \\ grant.
}

For correspondence: Dr G A Bernstein, Division of Child and Adolescent Psychiatry, Department of Psychiatry, University of Minnesota Medical School, F256/2B West, 2450 Riverside Avenue, Minneapolis MN 55454-1495, USA. Fax +1612273 9779.

Imipramine plus cognitive behavioural therapy $(\mathrm{Im}+\mathrm{CBT})$ v placebo plus $\mathrm{CBT}(\mathrm{Pl}+\mathrm{CBT})$ in school refusing adolescents

\begin{tabular}{lllll} 
Outcomes at 8 weeks & Im + CBT & $\begin{array}{l}\text { Placebo + } \\
\text { CBT }\end{array}$ & p Value & Effect size \\
\hline Mean weekly attendance & $70 \%$ & $28 \%$ & 0.02 & 0.29 \\
\hline CDRS-R score & 35 & 46 & 0.04 & 0.33 \\
& Im + CBT & $\begin{array}{l}\text { Placebo }+ \\
\text { CBT }\end{array}$ & RBI (95\% Cl) & NNT (CI) \\
\hline Met attendance goal & $54 \%$ & $17 \%$ & $210 \%(29$ to 383) & 3 (2 to 21) \\
\hline
\end{tabular}

$\neq C D R S-R=$ Children's Depression Rating Scale-Revised. Other abbreviations defined in glossary; RBI, NNT, and $\mathrm{Cl}$ calculated from data in article.

\section{COMMENTARY}

School refusal is a common, important clinical problem for which evidence-based treatments are lacking. ${ }^{1}$ Although the effect sizes are small to moderate in this pioneering study by Bernstein $e t$ al, the authors conclude appropriately that imipramine plus CBT is an effective treatment for school refusal in anxious depressed teenagers. Where does this leave the field?

Firstly, this study needs to be replicated in a more diverse sample of patients and providers before the result can be generalised to clinical practice. Secondly, evidence is emerging that CBT with a family component adds incremental benefit. ${ }^{2}$ Although the CBT protocol in this study included partial parent participation, an empirical test of greater parent involvement is desirable. Thirdly, although tricyclic antidepressants (TCAs) were the pharmacological treatment of choice 10 years ago, they have been supplanted by the selective serotonin reuptake inhibitors. ${ }^{3}$ Hence, the study needs replication with a change in medication. Finally, despite emerging evidence that TCAs may not be effective for MDD in youth, ${ }^{4}$ depression outcomes improved while anxiety outcomes did not. The meaning of this result is unclear, although older ages and measurement issues may have played a part. Quibbles aside, this important and welcome study provides empirical guidance about the treatment of severely ill children who refuse to attend school.

John S March, MD, MPH Duke University Medical Center Durham, North Carolina, USA

1 Elliott JG. School refusal: issues of conceptualisation, assessment, and treatment. J Child Psychol Psychiatry 1999;40:1001-12.

2 Cobham VE, Dadds MR, Spence SH. The role of parental anxiety in the treatment of childhood anxiety. J Consult Clin Psychol 1998;66:893-905.

3 March J. Pharmacotherapy of pediatric anxiety disorders: a critical review. In: D Beidel, editor. Treating anxiety disorders in youth: current problems and future solutions. Washington, DC: Anxiety Disorders Association of America, 1999.

4 Birmaher B. Should we use antidepressant medications for children and adolescents with depressive disorders? Psychopharmacol Bull 1998;34:35-9. 